\title{
Economía social en el turismo social. El caso de la asociación francesa VTF L'Esprit Vacances
}

\author{
Guerra Avalos, Eva Angélica * \\ https://orcid.org/0000-0002-3668-9765 \\ Reyes Uribe, Ana Cecilia* \\ https://orcid.org/0000-0001-9622-9448 \\ *Universidad de Guadalajara, México \\ E-mail: neotropia@gmail.com
}

Recibido: 16 de diciembre 2020

Aprobado: 22 de diciembre de 2020

\section{Resumen}

La economía social propone la primacía de las personas antes que las ganancias de capital. Es una alternativa al neoliberalismo integrada por entidades sin fines lucro creadas por la sociedad civil con un objetivo de beneficio colectivo. En Francia, las organizaciones de turismo social están fuertemente vinculadas a un pensamiento económico solidario, al propiciar el acceso justo y equitativo a las vacaciones, el bienestar humano y el desarrollo territorial. El objetivo de este artículo es evaluar la integración de las dimensiones y principios de la economía social y solidaria en el modelo de turismo social de la asociación francesa VTF L'Esprit Vacances (El Espíritu de Vacaciones). La investigación cualitativa interpretativa se basó en la metodología del estudio de casos y fue sustentada en el marco teórico de la economía social y solidaria. Se realizó una observación participante en cinco pueblos vacacionales de VTF. Los resultados muestran que las características del turismo social en VTF se interrelacionan armónicamente con la economía social y solidaria. Se concluye que el caso francés de economía social en el turismo social aporta bases para investigaciones posteriores sobre el acceso incluyente al turismo, la gestión socioeconómica solidaria y los valores humanos, culturales y ambientales.

Palabras clave: Economía social y solidaria, Turismo social, VTF, Francia. 


\title{
Social economy in social tourism. The case of the French association VTF L'Esprit Vacances
}

\begin{abstract}
The social economy proposes the primacy of people over capital gains. It is an alternative to neoliberalism, made up of non-profit entities created by civil society with an objective of collective benefit. In France, social tourism organizations are strongly linked to a solidarity-oriented economic thought, promoting fair and equitable access to vacations, human well-being and territorial development. The objective of this article is to evaluate the integration of the dimensions and principles of the social and solidarity-based economy in the social tourism model of the French association VTF L'Esprit Vacances (The Holidays Spirit). The qualitative interpretive research was based on a case study methodology and it was supported by the theoretical framework of the social and solidarity-based economy. A participant observation was conducted in five VTF holiday villages. The results show that the characteristics of social tourism in VTF are harmoniously interrelated with the social and solidarity-based economy. It is concluded that the French case of social economy in social tourism provides the basis for further research on inclusive access to tourism, solidarity socio-economic management and human, cultural and environmental values.
\end{abstract}

Keywords: Social and solidarity-based economy, social tourism, VTF, France.

\section{Introducción}

El neoliberalismo ha sido descrito por PuelloSocarrás (2013) como una expansión de los mercados globales donde imperan la acumulación del capital, la explotación, la dominación, la opresión y la alienación. Para Ortner (2011), la corriente neoliberal en el mundo ha tenido resultados polarizados entre las naciones ricas que amasan fortunas y aquellas donde el empobrecimiento de las personas ha sido desastroso. Coraggio (2011) explica que esta situación se ha debido a factores como la competencia feroz, los castigos impuestos a los que no pueden competir, la exclusión de empleos, el desgaste de salarios y el sector informal. Adicionalmente, Acosta (2011) argumenta que la libre competencia sin regulación de los mercados ha derivado en una inequidad social, en especulación financiera y en la violación de derechos económicos, sociales, culturales y ambientales.

Contrariamente, la economía social propone una realidad diferente a la capitalista que se fundamenta en alcanzar el buen vivir; es una alternativa para el desarrollo de una sociedad, sustentada en la convivencia humana, el reconocimiento de la diversidad cultural de cada país y la relación armónica con la naturaleza (Acosta, 2011). Se trata de una transición a otra economía construida colectivamente con estrategias sociales y políticas que dignifican la vida, independizan a los trabajadores, valoran la historia, las culturas e identidades distintas y reconocen los saberes (Coraggio, 2011). Así, la economía social se identifica en las dimensiones política, económica, social, humana, cultural y ecológica, en las que las entidades del sector trabajan con base en ciertos principios éticos.

En el contexto de la economía social se encuentran las organizaciones de turismo social, cuyo objetivo no es el lucro, sino garantizar mediante una gestión con enfoque solidario que el mayor número posible de personas, sin distinciones, tengan acceso a las vacaciones. A la vez, se busca detonar el desarrollo territorial, el intercambio sociocultural, la cohesión comunitaria y el desarrollo humano. VTF L'Esprit Vacances es una asociación francesa de turismo social que se desenvuelve en el sector de la economía social y solidaria.

El objetivo de este estudio es evaluar la integración de las dimensiones y principios de la economía social y solidaria en el modelo de turismo social de la asociación francesa VTF L'Esprit Vacances. 
La trabajo se expone en cuatro apartados: el marco teórico de la economía social y solidaria, la caracterización de las organizaciones de turismo social, la descripción del funcionamiento de turismo social en VTF y la discusión de las interrelaciones observadas.

\section{La economía social y solidaria}

Las nociones de la economía social se remontan a finales del siglo XIX en Francia para referirse a las asociaciones voluntarias de obreros y campesinos formadas en defensa de sus derechos sociales y económicos, frente a la explotación y el empobrecimiento causado por el capitalismo industrial. Los aportes pioneros a la economía social correspondieron a Charles Dunoyer, Constantin Pecquer, Fréderic Le Play, Charles Gide y Leon Walras. El término repuntó en la década de 1970 durante movimientos cooperativos, mutualistas $\mathrm{y}$ asociativos franceses que fortalecieron su reconocimiento institucional mediante la creación de un comité nacional, el cual aprobó en 1982 la Carta de la Economía Social. En el documento se reconoció el valor de una economía fundada en la solidaridad para resolver necesidades insatisfechas, sobre todo de las poblaciones con menos recursos (Borge y Li, 2015).

La economía social ha sido definida como un conjunto de entidades autónomas a los poderes públicos que brindan un servicio colectivo sin fines de lucro, están organizadas formalmente bajo un principio de adhesión voluntaria, funcionan con una gestión democrática e igualitaria en derechos y obligaciones de los socios, aplican los principios de solidaridad y de responsabilidad, y practican un régimen de propiedad y distribución de ganancias y excedentes en beneficio de la organización, los asociados y la sociedad, en el que priman las personas y el objetivo social antes que el capital (Ávila y Campos, 2018). La economía social se presenta a través de una diversidad de iniciativas que integran criterios éticos en las dimensiones política, económica, social, humana, cultural y ecológica, a lo largo de la cadena de producción, distribución, intercambio y consumo (Coheur, 2015).
En la economía social, la acción conjunta de personas, familias, comunidades y colectivos organizados en ámbitos locales, conlleva a la distribución de riqueza más equitativa y al desarrollo humano que incide en una mejor calidad de vida. La competencia puede regularse de forma transparente y se dan vínculos fraternales de trabajo cooperativo en libre asociación, sin privilegiar únicamente la utilidad económica. Las empresas tienen un rostro social que se despliega para todos los ciudadanos, incluyendo a sectores usualmente excluidos del trabajo (Coraggio, 2011). No se trata de una economía para pobres, sino de una propuesta de inclusión para el desarrollo de lazos sociales durante la satisfacción de necesidades y el ejercicio de derechos.

Las organizaciones de la economía social son privadas, pero se crean por la sociedad civil para dar respuesta a problemas y demandas sociales que no han sido resueltas ni por el sector público, ni por las empresas tradicionales basadas en la lógica del capital. Las formas organizativas incluyen a cooperativas, mutualidades, asociaciones, fundaciones y otras formas de empresa social que compartan las características de la economía social. Se identifican dos subsectores, uno que obtiene recursos de la venta de bienes y servicios sin fines de lucro, y otro de productores que suministran servicios de forma gratuita o a precios muy bajos. Se ha vinculado al Tercer Sector con las entidades de la economía social y las organizaciones no lucrativas (non-profit organizations), sin embargo, estas últimas no reúnen a iniciativas privadas que operan en el mercado, aún con una forma empresarial distinta al capitalista. De cualquier forma, ambas se ubican en el espacio entre la Economía Pública y la Economía Capitalista (Ávila y Campos, 2018).

Desde el año 2014, en Francia se ha ampliado el concepto a economía social y solidaria para reforzar su enfoque en el bienestar humano, y ha sido descrita como el conjunto de iniciativas socioeconómicas que priorizan la satisfacción de las necesidades de las personas y no el lucro, son independientes de los poderes públicos y se orientan por valores de equidad, solidaridad, sostenibilidad, participación, inclusión y compromiso con la comunidad. La 
economía social y solidaria promueve la mejora en la calidad de vida, el desarrollo territorial y el fortalecimiento del tejido social (Tapia y Alvarado, 2019). Los emprendimientos solidarios se han desarrollado de manera diferenciada a la clásica empresa capitalista (Vera y Ramírez, 2018).

La Ley 2014-856 (2014), relativa a la economía social y solidaria en Francia, establece que las organizaciones deben cumplir con al menos una de las siguientes condiciones: brindar apoyo a las personas en situación de fragilidad económica, social o personal, ya sean empleados, usuarios, miembros o beneficiarios; contribuir a la lucha contra la exclusión y las desigualdades, a la educación ciudadana, al desarrollo de lazos sociales y a la cohesión territorial; favorecer al desarrollo sostenible en sus dimensiones económica, social, ambiental y participativa, a la transición energética o a la solidaridad internacional.

Se infiere que los principales principios éticos compartidos por las organizaciones de la economía social y solidaria son: autonomía, autogestión, carácter no lucrativo, cooperación, solidaridad, trabajo, compromiso con el entorno, equidad, desarrollo humano y sostenibilidad ambiental (Acosta, 2011; Ávila y Campos, 2018; Coheur, 2015; Coraggio, 2011; Tapia y Alvarado, 2019).

\section{Organizaciones de turismo social en la economía social y solidaria}

Al turismo social pertenece cualquier organización turística, autónoma y formalizada, con un objetivo social diferente a la maximización de ganancias. Su finalidad es hacer accesibles los viajes y el turismo para un mayor número de personas. Este tipo de organizaciones se enmarcan en la economía social y solidaria (Caire, 2011). El término social evoca un sentido de fraternidad en la dinámica turística. También se le ha llamado turismo popular, turismo asociativo, turismo de base social o turismo no lucrativo. Particularmente en Francia, se ha utilizado la expresión turismo para todos -tourisme pour tous-.

El turismo ha sido uno de los escenarios del capitalismo enun procesoglobaldemovilizaciónentre fronteras. Grandes grupos turísticos internacionales se han expandido en sitios subdesarrollados para transformar recursos culturales y naturales en atractivos, con el apoyo del Estado en la apropiación y modificación del territorio (Palafox, 2013). Las empresas transnacionales buscan concesiones fiscales o legales del poder público en los países destino para alcanzar una alta rentabilidad, frente al deterioro territorial y de los recursos de la región anfitriona (Córdoba, 2014). Por otro lado, el acceso de las personas a las vacaciones puede volverse insostenible por los altos precios de un mercado en constante competencia.

En contraparte, en el turismo social los poderes públicos intervienen en conjunto con las organizaciones en economía social y solidaria, sustentadas en una gestión democrática, para detonar el desarrollo de las regiones y poner al alcance de los ciudadanos con menores recursos la posibilidad del bienestar a través de las relaciones familiares, la vida social y el ocio (Pyke, Pyke y Watuwa, 2019).

Las premisas del turismo social son aumentar la calidad de vida de grupos en situación vulnerable, disminuir prejuicios, reforzar lazos de familias, dignificar al ser humano y elevar el contacto cultural entre pueblos (Muñiz, 2001). La demanda se integra por colectivos de familias, jóvenes, adultos mayores, personas con discapacidad, obreros y emigrantes.

Caire (2011) explica que el proceso de integración de la economía social en el turismo social tiene sus antecedentes en Francia después de la Segunda Guerra Mundial. Tras el logro de las vacaciones pagadas en 1936, los hoteles no estaban adaptados en precios para recibir a familias de bajos ingresos y aún en 1945 la provisión de vacaciones familiares no era una prioridad del gobierno. Para cubrir esa ausencia de interés, activistas y voluntarios crearon casas de vacaciones familiares económicas en viejos hoteles, mansiones o castillos, donde las actividades y quehaceres estaban a cargo de los visitantes durante el verano, a quienes el sector público apoyaba con vales de las Cajas de Asignación Familiar (Caisses d'Allocations Familiales, CAF), un fondo de seguridad social. 
En 1958, la colaboración entre organizaciones y movimientos sociales dio origen a la construcción de los primeros pueblos vacacionales (village vacances) para todos, sin diferencia de clases, con el propósito de que expandieran los viajes y contribuyeran al desarrollo territorial; el precio de las estancias correspondía al salario mínimo, con una tarifa menor para niños. Con el aumento paulatino de la demanda en estos alojamientos, entre 1962 y 1965 el gobierno otorgó subsidios a las organizaciones y tarifas subvencionadas de transporte público. Los subsidios comenzaron a disminuir en los años 80 , dando paso a la subvención de la construcción de instalaciones y al apoyo directo al turista.

Una de esas ayudas está a cargo de la Agencia Nacional para los Cheques Vacacionales (Agence Nationale pour les Chèques-Vacances, ANCV). Estos cheques son distribuidos a agentes económicos, públicos y sociales para que se otorguen a sus empleados o beneficiarios conforme a su salario y puedan canjearse por servicios turísticos de alojamiento, transporte, alimentación y actividades de ocio con proveedores adheridos al programa. Mientras menor sea el salario más cheques se reciben y con estos se obtiene una rebaja entre el 5 y el 25 por ciento del precio de los servicios (ANCV, 2020).

Actualmente, son numerosos los agentes franceses privados y públicos que trabajan en reciprocidad, equidad y fraternidad para gestionar el turismo social: la administración central, las administraciones territoriales, sindicatos, cooperativas, mutualistas y asociaciones. Un ejemplo de organización de turismo social en economía social es la Unión Nacional de Asociaciones de Turismo (Union Nationale des Associations de Tourisme, UNAT), que pretende lograr cuatro principios fundamentales: garantizar el acceso a las vacaciones al mayor número de personas de diversos orígenes sociales y fomentar el intercambio social; enfatizar los valores humanistas y colectivos del turismo para el bienestar personal y la cohesión social; apoyar el desarrollo sostenible de los alojamientos turísticos, considerando el respeto de las características socioculturales y ambientales del destino; y lograr beneficios económicos mediante el apoyo de organizaciones sociales (UNAT, 2020). Entre las asociaciones que pertenecen a la UNAT se encuentra VTF (Vacances, Tourisme, Famille) L'Esprit Vacances.

\section{Metodología}

Se realizó una investigación cualitativa interpretativa con la metodología del estudio de casos. En ésta, se explora una situación en su contexto real, donde el investigador debe permanecer, familiarizarse, centrarse en interacciones personales y directas, identificar las relaciones dentro del sistema y buscar la comprensión de los resultados en su totalidad (Gutiérrez, Pozo y Fernández, 2002).

En un estudio de caso interpretativo una formulación teórica conduce la recolección y análisis de datos; las descripciones de información clave se utilizan para ilustrar, defender o desafiar teorías (Jiménez y Comet, 2016). De tal manera, primeramente, se elaboró un marco teórico de la economía social y solidaria. Así mismo, se caracterizaron las organizaciones de turismo social pertenecientes al sector de la economía social y solidaria.

La fase de trabajo in situ se llevó a cabo en junio 2015 durante una Misión Académica entre México y Francia organizada por la Asociación Mexicana de Centros de Enseñanza Superior en Turismo y Gastronomía (AMESTUR) y coordinada por la Asociación VTF. La recolección de datos sobre el funcionamiento del turismo social en VTF se hizo mediante la observación participante en cinco pueblos vacacionales de VTF: Les Bruyères y Le Domaine de Françon en la región de Aquitaine, Le Clair Canigou en Languedoc Rousillon, Le Castelet y Les Esquirousses en la región de Provence - Alpes Côte d'Azur (PACA). Se utilizaron las técnicas de diario de campo, entrevistas no estructuradas, reuniones con expertos, revisión de folletos, exploración del sitio web y registro fotográfico.

La intervención de las investigadoras se hizo en el rol de participantes en la misión académica y clientes de los complejos vacacionales. Los informantes clave fueron los coordinadores de la Misión Académica, trabajadores de los pueblos vacacionales, algunos huéspedes y representantes 
de AMESTUR, VTF, UNAT-PACA, ANVC y del Comité de Turismo Región PACA, con quienes se tuvo una reunión en Marsella.

\section{Resultados: modelo del turismo social en VTF}

VTF es una asociación independiente de turismo social que forma parte del sector de la economía social y solidaria. Fue fundada en 1956 en Thionville, Francia, por activistas sindicales, obreros y familiares que pugnaban por el derecho fundamental de las vacaciones para todos, bajo principios de precios solidarios, igualdad sociocultural y desarrollo personal de cada individuo En 1962 abrió su primera residencia vacacional en Alsacia (VTF, 2015).

Con una vocación enfocada principalmente en las familias y en grupos juveniles o de la tercera edad, VTF rechaza el ultraliberalismo y trabaja por una sociedad más justa e igualitaria donde se privilegie al ser humano, no sólo a las relaciones de capital. $\mathrm{Su}$ objetivo es que el turismo social sea reconocido como un componente del desarrollo individual y colectivo.

Como organización sin fines de lucro, VTF permite a las familias viajar según sus necesidades, medios e ingresos; estos últimos son la base para calcular las tarifas que se pagan. Ofrece precios justos y solidarios en la prestación de servicios. Además, hay gratuidad en la estancia de un niño menor de seis años por familia y para familias numerosas se ofrece un 50 por ciento de descuento a partir del tercer hijo y los siguientes, entre otras fórmulas más de descuento en los precios. Se reciben cheques vacacionales, bonos CAF y los afiliados de las comisiones obreras pueden beneficiarse de un descuento del 5 al 13 por ciento según los destinos elegidos y la temporada.

VTF no recibe subsidios públicos. Está a cargo de un consejo directivo constituido por voluntarios que supervisan el funcionamiento de la asociación. Administra 57 establecimientos de hospedaje -pueblos vacacionales, residencias y algunos bungalós-, la mayoría ubicados en la zona Sur del país. 25 complejos se localizan cerca del mar, 18 están en destinos de montaña y 14 en la campiña; 33 son propiedad de VTF y 24 pertenecen a sus socios colaboradores.

Los complejos son, según la propia asociación, de "talla media" y prescinden de lujos. Están alejados de grandes y masificados núcleos turísticos. La infraestructura permite la realización de actividades o la relajación, a elección de cada huésped. Las áreas comunes varían, pero son recurrentes las instalaciones para recepción, comedor, bar, deportes y usos múltiples. Pueden tener clubes de animación infantil y salas de televisión. 33 alojamientos tienen adecuaciones para personas con movilidad reducida. Los restaurantes proporcionan tres comidas al día, con un menú que incorpora gastronomía local.

Las habitaciones brindan el confort suficiente, son higiénicas y tienen camas cómodas. Se otorgan toallas, ropa de cama y papel higiénico, pero ninguna amenidad. No tienen teléfono y, por lo general, tampoco televisión, ni conexión a Internet. Con esto se pretende que quienes comparten el alojamiento dejen de lado la tecnología y vuelvan al diálogo.

La limpieza del alojamiento está a cargo de los huéspedes, a quienes se provee de lo necesario; es al final de las estancias cuando el personal asea las instalaciones. El precio es todo incluido en régimen de pensión completa. Se ofrecen también media pensión o sólo hospedaje. Se cubren vacaciones a lo largo del año; durante el invierno cobran popularidad los complejos vacacionales en entornos nevados para la práctica del esquí.

VTF pertenece a la UNAT y trabaja en una red asociativa con otros actores del turismo social, como colectividades locales, comités de empresas, mutualistas, sindicatos, las CAF y la ANVC. Participa corresponsablemente en proyectos para el desarrollo social.

La operación de VTF se garantiza con el trabajo de su personal, que se ciñe a una política de capacitación interna. La fuerza laboral es mayoritariamente de jóvenes, contratados aún cuando no cuenten con experiencia previa. El equipo base es limitado, por ello, se contratan asalariados temporales. En adición, 
se mantiene un convenio con AMESTUR para que estudiantes mexicanos de educación superior, adscritos a programas de turismo y gastronomía, realicen estancias de prácticas profesionales durante los periodos vacacionales de verano e invierno. Aunque hay puestos con labores específicas, algunos trabajadores son polivalentes, de modo que pueden atender la recepción, organizar un cóctel o hacer actividades de animación. Los directores se involucran en varias tareas; con este esquema de trabajo se alimenta el espíritu de cooperación. La política salarial es coherente con la responsabilidad social.

VTF se concentra en el turismo doméstico, al que atrae en su mercadotecnia $-i Y$ si nos quedamos en Francia durante las vacaciones?-. Los pueblos vacacionales se consideran servicios de interés económico general, con una misión social hacia el desarrollo local a través de la hospitalidad. Se establecen acciones participativas con entidades en diversas localidades, particularmente en las más aisladas y pequeñas, para dinamizar la economía con la venta de productos y servicios para la asociación o para los visitantes interesados en consumir particularidades culturales.

El modelo de VTF se dirige a todos los públicos, sin hacer distinciones ni exclusiones por condiciones religiosas, raciales, sociales, profesionales, físicas, de edad o de género. Promueve la mezcla social y el intercambio cultural, respetando las diferencias y la dignidad humana.

La animación sociocultural es un servicio esencial en VTF. Se basa en programas variados de actividades colectivas que buscan generar alegría, diversión, interacción social, desarrollo de aptitudes y conocimientos. Con juegos, deportes, excursiones, espectáculos, bailes, reuniones, entre otros, se logra un ambiente agradable de convivencia. Los animadores han sido capacitados en diplomados de especialización.

VTF apunta a una concepción no elitista de la cultura y a un turismo respetuoso de las comunidades. Da valor a la diversidad de identidades y al patrimonio, mediante la organización de paseos para que los huéspedes descubran el entorno, aprendan de él y convivan con los pobladores locales.

Las opciones de alojamiento se integran en armonía al ambiente natural y los descubrimientos territoriales permiten la inmersión en distintas geografías. Se procura evitar el gasto innecesario de recursos, por ejemplo, en la mayor parte de establecimientos se limitan los aparatos eléctricos en las habitaciones, se presta servicio de wifi sólo en ciertas áreas comunes o se apagan las luces de las instalaciones exteriores por la noche. Se han integrado energías renovables en algunas áreas de recepción. 


\section{Discusión}

Las dimensiones y principios de la economía social tienen una integración efectiva en el modelo de turismo social de VTF, como se muestra en la Tabla 1.

Tabla 1. Economía social en el modelo de turismo social de VTF.

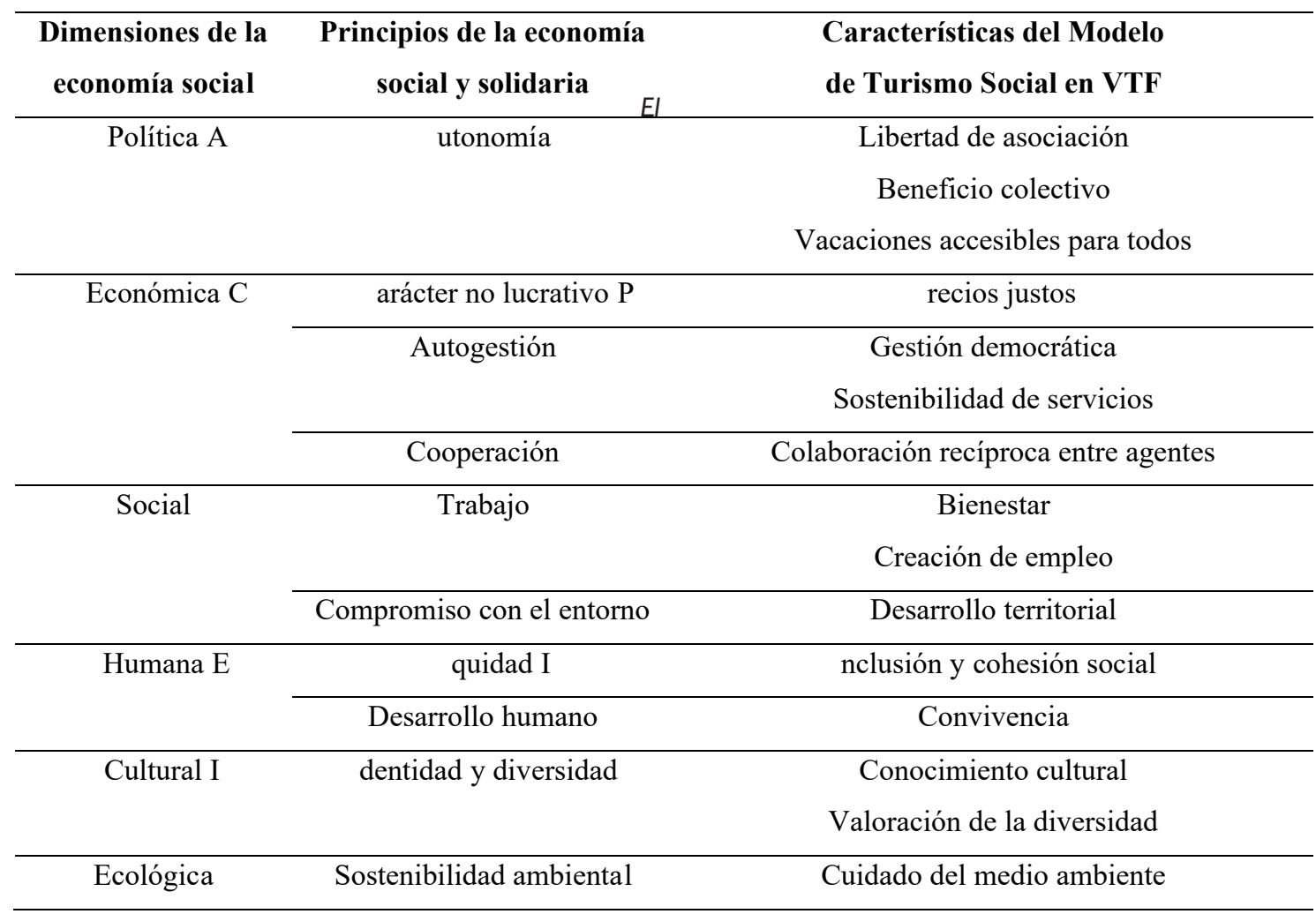

Elaboración propia.

En la dimensión política, VTF ejerce autonomía y libertad de asociación; se ha convertido en un agente de transformación social a través de un turismo solidario y más humano. En la dimensión económica, la asociación contribuye al crecimiento de la economía en beneficio de los ciudadanos. $\mathrm{Su}$ carácter no lucrativo ayuda a las familias y grupos menos favorecidos, por medio de tarifas diferenciadas y decrecientes adaptadas a los ingresos y necesidades de cada colectivo; ofrece diferentes opciones de reducción de precios. Sus mecanismos de autogestión hacen de VTF un proyecto económicamente viable y sostenible, con servicios diversificados en múltiples regiones de Francia y en cada estación del año. El trabajo en redes de cooperación mantiene relaciones corresponsables en el desarrollo de los espacios sociales.

Respecto a la dimensión social, la economía social ha sido una plataforma para que VTF coadyuve en mejorar la calidad de vida de las personas. Destacan la creación de puestos de trabajo en los complejos vacacionales y la igualdad de oportunidades laborales. El compromiso de VTF con el entorno social, mediante la dinamización de economías locales en diversos territorios, valida su participación en desarrollo local sostenible y comunitario.

Para la dimensión humana, la práctica ética de la equidad en la recepción de todo tipo de personas impulsa los derechos humanos y los valores sociales. 
La animación es una pieza capital que conduce al desarrollo de las personas al motivarlas a sentirse felices y relacionarse en armonía con los demás. La puesta en valor de la diversidad y el patrimonio, en la dimensión cultural, refuerza la identidad francesa. Finalmente, en la dimensión ecológica, VTF participa en la preservación de la sostenibilidad ambiental, pues reduce el impacto del consumo energético con medidas de austeridad.

\section{Conclusiones}

Se ha evidenciado que la economía social se interrelaciona armónicamente con el turismo social. La interpretación permite analizar de qué manera se alinean las dimensiones de la economía social con los principios de la economía social y solidaria, y cómo ambos parámetros se integran en el funcionamiento del turismo social en la organización VTF L'Esprit Vacances. La limitación más significativa del estudio fue la recolección de datos en únicamente cinco de los 57 establecimientos administrados por VTF, no obstante, se cotejó con los informantes clave que el modelo de turismo social observado es aplicable a la totalidad de los pueblos y residencias vacacionales. Es importante resaltar que actualmente la asociación opera con las mismas características reportadas en los resultados.

La capacidad de Francia de garantizar a sus ciudadanos el acceso justo e incluyente a las vacaciones, sin ningún tipo de discriminación y con atención especial en los colectivos más vulnerables, es un referente a considerar para la investigación de la economía social y el turismo social en el contexto de otras naciones. El criterio de solidaridad para el beneficio común, alejado de la lógica capitalista, abre oportunidades para indagar formas de gestión económica, cooperación y compromiso social conducentes a una mejor calidad de vida de las personas y al desarrollo territorial, bajo una óptica de fraternidad donde el ganar - ganar en el turismo es posible. Además, puede explorarse con mayor profundidad el reencuentro con los valores humanos, las identidades culturales y los espacios naturales propuesto por la economía y el turismo social.

\section{Agradecimientos}

Gracias a la AMESTUR por la organización de la Misión Académica México - Francia; a VTF y UNAT - Región PACA por facilitar el trabajo de campo y otorgar el alojamiento en pueblos vacacionales, los alimentos y la transportación terrestre; a la Universidad de Guadalajara (UDG) por el financiamiento del transporte aéreo internacional a través del PROFOCIE. Se reconoce especialmente el apoyo de José Luis Santana Medina (UDG), José Luis Isidor Castro (AMESTUR), Michael Gallien (VTF) y Salvador Gómez Nieves (UDG).

\section{Referencias bibliográficas}

Acosta, A. (2011). La economía social y solidaria en el centro del debate. Un aporte sustantivo desde la economía del trabajo. En J. L. Coraggio, Economía social y solidaria. El trabajo antes que el capital (pp. 9-32). Quito, Ecuador: Ediciones Abya-Yala.

ANCV (2020). Parce que les vacances, c'est essentiel. Recuperado de: https://www.ancv. com/

Ávila, R. C. y Campos, J. L. (2018). La economía social ante los paradigmas económicos emergentes: Innovación social, economía colaborativa, economía circular, responsabilidad social empresarial, economía del bien común, empresa social y economía solidaria. CIRIECEspaña, Revista de Economía Publica, Social y Cooperativa (93), 5-50. doi:10.7203/ CIRIEC-E.93.12901

Borge, D. y Li, F. (2015, septiembre). Economía social, economía solidaria y economía laboral: un abordaje comparativo desde sus perspectivas conceptuales. IX Congreso RULESCOOP, La Plata, Argentina. http://sedici.unlp.edu.ar/ handle/10915/50006

Caire, G. (2011). Social Tourism and the Social Economy. En S. McCabe, L. Minnaert \& A. Diekmann (Eds.) Social Tourism in Europe Theory and Practice (pp. 73-88). Bristol, UK: 
Channel View Publications.

Coheur, A. (2015). Libro Blanco: La Economía Social... retoma la iniciativa. Propuestas para hacer de la Economía Social un pilar de la Unión Europea. Bruselas, Bélgica: Social Economy Europe.

Coraggio, J.L. (2011). Economía social y solidaria. El trabajo antes que el capital. Quito, Ecuador: Ediciones Abya-Yala.

Córdoba Hernández, R. (2014). El modelo de urbanismo neoliberal enfocado en el turismo y el ocio como ejemplo de insostenibilidad. Encrucijadas. Revista Crítica de Ciencias Sociales (8), 189-203. https://dialnet.unirioja. es/servlet/articulo? codigo $=4999338$

Gutiérrez Pérez, J., Pozo Llorente, T. y Fernández Cano, A. (2002). Los estudios de caso en la lógica de la investigación interpretativa. Arbor, 171(675), 533-557. https://doi.org/10.3989/ arbor.2002.i675.1045

Jiménez Chaves, V.E.y Comet Weiler, C.(2016). Los estudios de casos como enfoque metodológico. Academo, Revista de Investigación en Ciencias Sociales y Humanidades, 3(2). https://dialnet. unirioja.es/servlet/articulo?codigo $=5757749$

Ley 2014-856 (2014). Loi 2014-856 du 31 juillet 2014 relative à l'économie sociale et solidaire. Journal Officiel de la République Française 0176. https://www.legifrance.gouv.fr/jorf/id/ JORFTEXT000029313296/

Muñiz Aguilar, D. (2001). La política de turismo social. Andalucía, España: Junta de Andalucía.

Ortner, S. Sobre el neoliberalismo (R. Llanes y A. Horta, Trad.) (2015). Antrópica: Revista de Ciencias Sociales y Humanidades, 1(1), 126-135. https://dialnet.unirioja.es/servlet/ articulo? codigo $=7402365$

Palafox Muñoz, A. (2013). El turismo como eje de acumulación. Nómadas. Revista crítica de
Ciencias Sociales y Jurídicas, 161-174. http:// dx.doi.org/10.5209/rev_NOMA.2013.42347.

Puello-Socarrás, J.F. (2013) Ocho tesis sobre el Neoliberalismo (1973-2013). En H. Ramírez (Ed.) (pp. 13-57). O neoliberalismo sul-americano em clave transnacional: enraizamento, apogeu e crise. São Leopoldo, Brasil: Oikos.

Pyke, J., Pyke, S. y Watuwa, R. (2019). Social tourism and well-being in a first nation community. Annals of Tourism Research (77), 38-48. doi:10.1016/j.annals.2019.04.013

Tapia-Toral, M.C. y Alvarado-Espinoza, F.G. (2019). Principios básicos de la economía social y solidaria en el marco de la satisfacción de las necesidades humanas colectivas. Dominio de las Ciencias, 5(3), 731-740. http://dx.doi. org/10.23857/dc.v5i3.961

UNAT (2020). Tourisme Social et Solidaire. Recuperado de: https://www.unat.asso.fr/

Vera Peña, V. M. y Ramírez Frías, C. H. (2018). La economía solidaria para el desarrollo del turismo comunitario en la Isla Santay. Universidad y Sociedad, 10(3), 159-164. https://rus.ucf.edu. cu/index.php/rus/article/view/900/990

VTF (2015). L'Esprit VTF Vacances. Recuperado de: https://www.vtf-vacances.com/fr 